\title{
Effect of Age on Laparoscopic Surgery and Postoperative Chemotherapy in Elderly Patients With Colorectal Cancer
}

\author{
Hyun Hee Kim, Myong Hoon Ihn, Yun Hee Lee, Jihyoun Lee, Sangchul Yun, Sung Woo Cho \\ Department of Surgery, Soonchunhyang University Seoul Hospital, Soonchunhyang University College of Medicine, Seoul, Korea
}

Purpose: We aimed to evaluate the postoperative complications of laparoscopic colorectal cancer (CRC) surgery and the adverse events of postoperative chemotherapy in elderly patients compared to younger patients and to identify the factors influencing the termination of postoperative chemotherapy.

Methods: Between June 2015 and May 2018, 188 patients with CRC underwent laparoscopic surgery with curative intent. Patients aged $\geq 70$ were defined as elderly. Postoperative complications and adverse events of chemotherapy were assessed by using the Clavien-Dindo classification and the Common Terminology Criteria for Adverse Events, respectively. The clinicopathological factors were analyzed retrospectively.

Results: Seventy-eight patients were considered elderly with a mean age of $77.5 \pm 5.5$ years. Overall postoperative complications occurred in 68 patients (36.2\%). Age and primary tumor location were independent predictors of overall postoperative complications. Smoking history was the only independent predictor of major postoperative complications. Of 113 patients who were recommended postoperative chemotherapy, 90 patients $(79.6 \%)$ received postoperative chemotherapy. Overall adverse events occurred in 40 patients (44.4\%). The American Society of Anesthesiologists physical status classification and chemotherapy regimen were significantly associated with overall adverse events. The chemotherapy regimen was the only factor significantly associated with severe adverse events. Of 90 patients, postoperative chemotherapy could not be completed in $11(12.2 \%)$. Age was the only factor significantly associated with stopping postoperative chemotherapy $(\mathrm{P}=0.003)$.

Conclusion: This study shows that laparoscopic CRC surgery and postoperative chemotherapy were feasible in elderly patients. Further efforts are needed to ensure that elderly patients have the opportunity to make informed decisions regarding postoperative chemotherapy.

\section{Keywords: Elderly patients; Age; Colorectal cancer; Laparoscopic surgery; Adjuvant chemotherapy}

\section{INTRODUCTION}

Colorectal cancer (CRC) is a major cause of morbidity and mortality in the elderly because more than $70 \%$ of the cases occur in people over 65 years of age [1]. Furthermore, its incidence is ex-

Received: Aug 30, 2019 - Revised: Sep 23, 2019 - Accepted: Oct 3, 2019 Correspondence to: Myong Hoon Ihn, M.D.

Department of Surgery, Soonchunhyang University Seoul Hospital, Soonchunhyang University College of Medicine, 59 Daesagwan-ro, Yongsan-gu, Seoul 04401, Korea

Tel: +82-2-710-3234, Fax: +82-2-710-3088

E-mail: income78@hanmail.net

ORCID: https://orcid.org/0000-0002-9522-401X

(C) 2020 The Korean Society of Coloproctology

This is an open-access article distributed under the terms of the Creative Commons Attribution NonCommercial License (https://creativecommons.org/licenses/by-nc/4.0) which permits unrestricted noncommercial use, distribution, and reproduction in any medium, provided the original work is properly cited. pected to increase by $60 \%$ by 2030 [2]. With increases in life expectancy, the number of elderly patients who need treatment for CRC will also increase.

Surgical intervention is the optimal treatment for patients with resectable CRC. Laparoscopic CRC surgery has advantages, such as less postoperative pain, quicker return of bowel function, shorter hospital stays, better cosmetic effects, and lower incidence of postoperative complications, compared to open CRC surgery $[3,4]$. In addition, several randomized trials have demonstrated that laparoscopic CRC surgery provided equivalent oncologic results compared to open CRC surgery $[4,5]$. Therefore, laparoscopic CRC surgery appears to be a suitable treatment for elderly patients. However, during laparoscopic surgery, pneumoperitoneum and changing position affect hemodynamics and the respiratory system, particularly in elderly patients [6]. Furthermore, elderly patients often exhibit more comorbidities than younger pa- 
tients, which could contribute to the increase in postoperative morbidity and mortality in elderly patients $[1,7]$. Therefore, there is still conflicting evidence regarding the safety and benefits of laparoscopic CRC surgery for elderly patients.

In addition, postoperative chemotherapy for CRC has been used in the adjuvant setting for stage III and high-risk stage II patients and is administered to patients with metastatic disease [8]. The use of postoperative chemotherapy in elderly patients, however, remains controversial due to concerns over both toxicity and death from causes unrelated to cancer. Although several studies have suggested that advanced age alone should not be used to exclude patients from effective treatment $[9,10]$, elderly patients are significantly less likely to be referred for and receive guidelinerecommended postoperative chemotherapy $[11,12]$.

Therefore, the aim of this study was to evaluate the postoperative complications of laparoscopic CRC surgery and the adverse events of postoperative chemotherapy in elderly patients compared to younger patients, and to identify the factors influencing the termination of postoperative chemotherapy.

\section{METHODS}

Consecutive patients who underwent laparoscopic surgery for CRC at our institution between June 2015 and May 2018, were retrospectively analyzed. The study included patients with tumor stages I, II, or III, as well as patients with metastatic disease who underwent surgery with curative intent (R0 resection). Patients were excluded if they underwent conventional open surgery or nonradical surgery, if there was evidence of concurrent malignancy, or if surgery was performed with palliative intent. This study was approved by the Institutional Review Board at the Soonchunghyang University Seoul Hospital (2019-08-019). Informed consent was waived according to the institutional rules for retrospective studies.

Clinicopathological factors, including patient age, sex, body mass index, previous operative history, medical history, the American Society of Anesthesiologists (ASA) physical status classification, the Charlson Comorbidity Index (CCI), primary tumor location, operative procedure, open conversion, operative time, estimated blood loss, the length of hospital stay, postoperative morbidity, mortality within 30 days following surgery, postoperative chemotherapy, chemotherapy regimen, chemotherapy dose, and mortality within 1 year following surgery were recorded after surgery.

Elderly age was defined as age 70 years or older, as in a previous study [13]. The primary tumor location was divided into the colon and the rectum. Tumor stage was classified by using the 8th edition of the American Joint Committee on Cancer staging system. Laparoscopic surgery was performed in all participants and the surgeon decided whether to perform a right or left hemicolectomy, anterior resection, low/ultra-low anterior resection, or colostomy procedure including abdominoperineal resection and
Hartmann operation.

Operative mortality was defined as death within 30 days of surgery. Postoperative complications were accessed by using the Clavien-Dindo (CD) classification [14]. Overall postoperative complications were defined as those with CD grade II or higher, and complications with $\mathrm{CD}$ grades $0-\mathrm{I}$ were defined as unremarkable recovery. Major postoperative complications were defined as those with $\mathrm{CD}$ grade III or higher, and minor postoperative complications were defined as those with CD grades $0-\mathrm{II}$, as in a previous study [15]. Anastomotic complications, including anastomotic leakage or an intraabdominal abscess, were detected by CT scanning. Postoperative ileus was defined as the absence of intestinal function for 72 hours or more after surgery and was confirmed by plain radiography. Urinary retention was defined as the inability to self-void after removing the urinary catheter, requiring catheterization or reinsertion of an indwelling catheter.

Standard postoperative chemotherapies were administered in accordance with the National Comprehensive Cancer Network $(\mathrm{NCCN})$ guidelines [8]. Adverse events of postoperative chemotherapy were accessed by using the Common Terminology Criteria for Adverse Events (CTCAE) version 5.0 [16]. Overall adverse events were defined as those with CTCAE grade II or higher, and adverse events with CTCAE grades 0-I were defined as an uneventful course. Severe adverse events were defined as those with CTCAE grade III or higher, and mild adverse events were defined as those with CTCAE grades 0-II, as in a previous study [17].

Categorical variables were analyzed using the chi-squared test or Fisher exact test. Continuous variables were compared using Student t-tests. Variables with P-values $\leq 0.1$ in univariate analyzes were included in multivariate logistic regression to identify factors that were independently and significantly associated with postoperative complications, adverse events of postoperative chemotherapy, and withdrawal of postoperative chemotherapy. P-values $\leq$ 0.05 were considered statistically significant. Statistical analyzes were performed with the use of SPSS ver. 18.0 (SPSS Inc., Chicago, IL, USA).

\section{RESULTS}

Between June 2015 and May 2018, 230 patients underwent CRC surgery at our hospital. Of these, 188 patients were included in the study. A total of 42 patients were excluded because of conventional open surgery in 9 patients, transanal local excision in 14 patients, concurrent malignancy in 2 patients, and palliative surgery in 17 patients.

Baseline characteristics and postoperative outcomes are summarized in Table 1. There were no significant differences in baseline characteristics between the elderly patients and nonelderly patients, except for gender, smoking and alcohol history, hypertension, and ASA physical status classification. The mean age of the patients was $66.3 \pm 11.8$ years. The mean age of patients in the nonelderly and elderly groups were $58.4 \pm 8.1$ and $77.5 \pm 5.5$ years, 
Table 1. Patient characteristics and postoperative outcomes $(n=188)$

\begin{tabular}{|c|c|c|c|}
\hline Characteristic & $\begin{array}{c}\text { Nonelderly } \\
(\mathrm{n}=110)\end{array}$ & $\begin{array}{l}\text { Elderly } \\
(n=78)\end{array}$ & P-value \\
\hline Age (yr) & $58.4 \pm 8.1$ & $77.5 \pm 5.5$ & $<0.001$ \\
\hline Sex & & & 0.007 \\
\hline Male & $69(62.7)$ & $33(42.3)$ & \\
\hline Female & $41(37.3)$ & $45(57.7)$ & \\
\hline Body mass index $\left(\mathrm{kg} / \mathrm{m}^{2}\right)$ & $23.4 \pm 2.9$ & $24.1 \pm 3.8$ & 0.172 \\
\hline Primary tumor location & & & 0.100 \\
\hline Colon & $60(54.5)$ & $52(66.7)$ & \\
\hline Rectum & $50(45.5)$ & $26(33.3)$ & \\
\hline Operative procedure & & & 0.223 \\
\hline Hemicolectomy & 37 (33.6) & $38(48.7)$ & \\
\hline Anterior resection & $23(20.9)$ & $14(17.9)$ & \\
\hline LAR/ultra-LAR & $44(40.0)$ & $23(29.5)$ & \\
\hline Colostomy & $6(5.5)$ & $3(3.8)$ & \\
\hline Open conversion & & & 0.280 \\
\hline No & $107(97.3)$ & 73 (93.6) & \\
\hline Yes & $3(2.7)$ & $5(6.4)$ & \\
\hline Operative time (min) & $280.2 \pm 115.1$ & $259.0 \pm 88.3$ & 0.174 \\
\hline Estimated blood loss (mL) & $181.7 \pm 137.3$ & $209.0 \pm 309.6$ & 0.414 \\
\hline Previous operative history & & & 0.144 \\
\hline No & $83(75.5)$ & $51(65.4)$ & \\
\hline Yes & $27(24.5)$ & $27(34.6)$ & \\
\hline Smoking history & & & 0.016 \\
\hline No & $69(62.7)$ & $62(79.5)$ & \\
\hline Yes & 41 (37.3) & $16(20.5)$ & \\
\hline Alcohol history & & & 0.001 \\
\hline No & $65(59.1)$ & $64(82.1)$ & \\
\hline Yes & $45(40.9)$ & $14(17.9)$ & \\
\hline Hypertension & & & 0.001 \\
\hline No & 75 (68.2) & $33(42.3)$ & \\
\hline Yes & 35 (31.8) & $45(57.7)$ & \\
\hline Diabetes & & & 0.053 \\
\hline No & $91(82.7)$ & 55 (70.5) & \\
\hline Yes & 19 (17.3) & 23 (29.5) & \\
\hline ASA PS classification & & & 0.003 \\
\hline I & $33(30.0)$ & $8(10.3)$ & \\
\hline$\|$ & $66(60.0)$ & $62(79.5)$ & \\
\hline III-IV & $11(10.0)$ & 8 (10.3) & \\
\hline
\end{tabular}

(Continued to the next)
Table 1. Continued

\begin{tabular}{|c|c|c|c|}
\hline Characteristic & $\begin{array}{l}\text { Nonelderly } \\
(n=110)\end{array}$ & $\begin{array}{l}\text { Elderly } \\
(n=78)\end{array}$ & P-value \\
\hline Charlson Comorbidity Index & & & 0.091 \\
\hline 0 & 75 (68.2) & $40(51.3)$ & \\
\hline 1 & $23(20.9)$ & $28(35.9)$ & \\
\hline 2 & $9(8.2)$ & $8(10.3)$ & \\
\hline$\geq 3$ & $3(2.7)$ & $2(2.6)$ & \\
\hline T stage & & & 0.052 \\
\hline $0-2$ & $51(46.4)$ & $25(32.1)$ & \\
\hline $3-4$ & $59(53.6)$ & $53(67.9)$ & \\
\hline N stage & & & 1.000 \\
\hline 0 & $63(57.3)$ & $44(56.4)$ & \\
\hline $1-2$ & $47(42.7)$ & $34(43.6)$ & \\
\hline Tumor stage & & & 0.307 \\
\hline $0-1$ & $38(34.5)$ & $18(23.1)$ & \\
\hline 2 & $25(22.7)$ & $25(32.1)$ & \\
\hline 3 & $42(38.2)$ & $31(39.7)$ & \\
\hline 4 & $5(4.5)$ & $4(5.1)$ & \\
\hline Histology of primary tumor & & & 0.303 \\
\hline Well & $23(20.9)$ & $14(17.9)$ & \\
\hline Moderately & 81 (73.6) & 55 (70.5) & \\
\hline Mucinous or poorly & $6(5.5)$ & $9(11.5)$ & \\
\hline Lymphatic invasion & & & 0.096 \\
\hline No & $72(65.5)$ & $41(52.6)$ & \\
\hline Yes & 38 (34.5) & $37(47.4)$ & \\
\hline Venous invasion & & & 0.126 \\
\hline No & $81(73.6)$ & 49 (62.8) & \\
\hline Yes & $28(25.5)$ & 29 (37.2) & \\
\hline Perineural invasion & & & 1.000 \\
\hline No & $88(80.0)$ & $60(76.9)$ & \\
\hline Yes & $22(20.0)$ & $18(23.1)$ & \\
\hline Length of hospital stay (day) & $12.17 \pm 7.1$ & $15.0 \pm 9.3$ & 0.024 \\
\hline Clavien-Dindo classification grade & & & 0.001 \\
\hline 0 & $61(55.5)$ & $20(25.6)$ & \\
\hline । & 18 (16.4) & $21(26.9)$ & \\
\hline$\|$ & $24(21.8)$ & 30 (38.5) & \\
\hline III & $7(6.4)$ & $7(9.0)$ & \\
\hline IV-V & $0(0)$ & $0(0)$ & \\
\hline Reoperation & $3(2.7)$ & $5(6.4)$ & 0.280 \\
\hline 30-Day mortality & $0(0)$ & $0(0)$ & - \\
\hline
\end{tabular}

Values are presented as mean \pm standard deviation or number (\%).

LAR, low anterior resection; ASA PS, American Society of Anesthesiologists physical status. 
respectively. A total of 33 elderly patients (42.3\%) were male. We performed rectal cancer surgery in 76 patients $(40.4 \%)$, including low/ultra-low anterior resection in 67 patients (35.6\%), and colostomy procedure in 9 patients (4.8\%). A total of 8 patients (4.2\%) were converted to conventional open surgery. In the postoperative outcomes, a significantly longer length of hospital stay characterized the elderly patients compared with the nonelderly pa- tients. There was a significant difference in $\mathrm{CD}$ grade between the elderly patients and nonelderly patients $(\mathrm{P}=0.001)$. Eight patients (4.3\%) underwent reoperations. There was no patient mortality within 30 days after surgery.

Postoperative complications occurred in 164 cases, including 70 (37.2\%) in grade I, $80(44.8 \%)$ in grade II, $6(3.2 \%)$ in grade IIIa, and $8(4.2 \%)$ in grade IIIb, respectively. The most common com-

Table 2. Univariate and multivariate analysis of factors affecting overall postoperative complications after laparoscopic CRC surgery ( $\mathrm{n}=188$ )

\begin{tabular}{|c|c|c|c|c|c|c|}
\hline Characteristic & $\begin{array}{l}\text { Unremarkable recovery } \\
\qquad(n=120)\end{array}$ & $\begin{array}{l}\text { Overall complication } \\
\qquad(\mathrm{n}=68)\end{array}$ & P-value & $\mathrm{OR}$ & $95 \% \mathrm{Cl}$ & P-value \\
\hline Age (yr) & & & 0.009 & & & 0.003 \\
\hline Nonelderly & 79 (65.8) & $31(45.6)$ & & 1 & & \\
\hline Elderly & $41(34.2)$ & $37(54.4)$ & & 2.608 & $1.386-4.905$ & \\
\hline Mean $\pm S D$ & $64.7 \pm 12.1$ & $69.1 \pm 10.8$ & 0.014 & & & \\
\hline Sex & & & 0.762 & & & \\
\hline Male & $64(53.3)$ & $38(55.9)$ & & & & \\
\hline Female & $56(46.7)$ & $30(44.1)$ & & & & \\
\hline Body mass index $\left(\mathrm{kg} / \mathrm{m}^{2}\right)$ & $23.8 \pm 3.0$ & $23.4 \pm 3.7$ & 0.371 & & & \\
\hline Primary tumor location & & & 0.046 & & & 0.017 \\
\hline Colon & $78(65.0)$ & $34(50.0)$ & & 1 & & \\
\hline Rectum & $42(35.0)$ & $34(50.0)$ & & 2.170 & $1.151-4.094$ & \\
\hline Operative procedure & & & 0.264 & & & \\
\hline Hemicolectomy & $50(41.7)$ & $25(36.8)$ & & & & \\
\hline Anterior resection & $27(22.5)$ & $10(14.7)$ & & & & \\
\hline LAR/ultra-LAR & 39 (32.5) & $28(41.2)$ & & & & \\
\hline Colostomy & $4(3.3)$ & $5(7.4)$ & & & & \\
\hline Open conversion & & & 0.140 & & & \\
\hline No & $117(97.5)$ & $63(92.6)$ & & & & \\
\hline Yes & $3(2.5)$ & $5(7.4)$ & & & & \\
\hline Operative time (min) & $265.1 \pm 104.7$ & $282.5 \pm 105.6$ & 0.276 & & & \\
\hline Estimated blood loss (mL) & $169.5 \pm 135.0$ & $234.6 \pm 325.8$ & 0.120 & & & \\
\hline Previous operative history & & & 1.000 & & & \\
\hline No & $85(70.8)$ & $49(72.1)$ & & & & \\
\hline Yes & 35 (29.2) & $19(27.9)$ & & & & \\
\hline Smoking history & & & 0.509 & & & \\
\hline No & $86(71.7)$ & 45 (66.2) & & & & \\
\hline Yes & 34 (28.3) & 23 (33.8) & & & & \\
\hline Alcohol history & & & 0.871 & & & \\
\hline No & 83 (69.2) & 46 (67.6) & & & & \\
\hline Yes & 37 (30.8) & $22(32.4)$ & & & & \\
\hline Hypertension & & & 0.543 & & & \\
\hline No & 71 (59.2) & $37(54.4)$ & & & & \\
\hline Yes & 49 (40.8) & 31 (45.6) & & & & \\
\hline
\end{tabular}


plication was mild electrolyte imbalance, but that spontaneously resolved in all patients without specific treatment. Among postoperative complications with CD grade III, intraabdominal abscess, anastomotic leakage, and bleeding occurred in 6 patients (3.2\%), 6 patients $(3.2 \%)$, and 2 patients (1.1\%), respectively. Of these, revision surgery due to anastomotic leakage was performed in 6 patients $(3.2 \%)$ and bleeder ligation due to postoperative bleeding was performed in 2 patients (1.1\%). A percutaneous drainage catheter was inserted in 6 patients (3.2\%) due to intraabdominal abscess (Supplementary Table 1).

Overall postoperative complications occurred in 68 patients (36.2\%). We found significant differences in overall postoperative complications according to the age and primary tumor location in univariate analysis. In multivariate analysis, age (odds ratio
[OR], 2.608; 95\% confidence interval [CI], 1.386 to 4.905 ; $\mathrm{P}=0.003)$ and primary tumor location $(\mathrm{OR}, 2.170 ; 95 \% \mathrm{CI}, 1.151$ to $4.094 ; \mathrm{P}=0.017$ ) were independent predictors of overall postoperative complications after laparoscopic CRC surgery (Table 2). Major postoperative complications occurred in 14 patients (7.4\%). Compared to minor postoperative complications, operative time and smoking history were significantly different in univariate analysis. However, age was not significantly different between minor and major postoperative complications after laparoscopic CRC surgery $(\mathrm{P}=0.578)$. In multivariate analysis, only smoking history was an independent predictor of major postoperative complications (OR, 1.785; 95\% CI, 1.003 to $3.178 ; \mathrm{P}=$ 0.049) (Table 3).

Of 188 consecutive patients, 113 patients $(60.1 \%)$ were recom-

Table 2. Continued

\begin{tabular}{|c|c|c|c|c|c|c|}
\hline Characteristic & $\begin{array}{l}\text { Unremarkable recovery } \\
\qquad(n=120)\end{array}$ & $\begin{array}{l}\text { Overall complication } \\
\qquad(\mathrm{n}=68)\end{array}$ & P-value & $\mathrm{OR}$ & $95 \% \mathrm{Cl}$ & P-value \\
\hline Diabetes & & & 0.363 & & & \\
\hline No & $96(80.0)$ & $50(73.5)$ & & & & \\
\hline Yes & $24(20.0)$ & 18 (26.5) & & & & \\
\hline ASA PS classification & & & 0.240 & & & \\
\hline । & $28(23.3)$ & $13(19.1)$ & & & & \\
\hline$\|$ & 77 (64.2) & $51(75.0)$ & & & & \\
\hline III-IV & 15 (12.5) & $4(5.9)$ & & & & \\
\hline Charlson Comorbidity Index & & & 0.760 & & & \\
\hline 0 & $74(61.7)$ & $41(60.3)$ & & & & \\
\hline 1 & $33(27.5)$ & 18 (26.5) & & & & \\
\hline 2 & $11(9.2)$ & $6(8.8)$ & & & & \\
\hline$\geq 3$ & $2(1.7)$ & $3(4.4)$ & & & & \\
\hline T stage & & & 0.122 & & & \\
\hline $0-2$ & $54(45.0)$ & $22(32.4)$ & & & & \\
\hline $3-4$ & $66(55.0)$ & $46(67.6)$ & & & & \\
\hline N stage & & & 0.445 & & & \\
\hline 0 & $71(59.2)$ & $36(52.9)$ & & & & \\
\hline $1-2$ & 49 (40.8) & $32(52.9)$ & & & & \\
\hline Tumor stage & & & 0.221 & & & \\
\hline $0-1$ & $41(34.2)$ & $15(22.1)$ & & & & \\
\hline 2 & $30(25.0)$ & $20(29.4)$ & & & & \\
\hline 3 & $42(35.0)$ & 31 (45.6) & & & & \\
\hline 4 & $7(5.8)$ & $2(2.9)$ & & & & \\
\hline Histology of primary tumor & & & 0.238 & & & \\
\hline Well & $26(21.7)$ & $11(16.2)$ & & & & \\
\hline Moderately & $82(68.3)$ & $54(79.4)$ & & & & \\
\hline Mucinous or poorly & $12(10.0)$ & $3(4.4)$ & & & & \\
\hline
\end{tabular}

Values are presented as mean \pm standard deviation or number (\%).

$\mathrm{OR}$, odds ratio; Cl, confidence interval; LAR, low anterior resection; ASA PS, American Society of Anesthesiologists physical status. 
Table 3. Univariate and multivariate analysis of factors affecting major postoperative complications after laparoscopic CRC surgery ( $\mathrm{n}=188$ )

\begin{tabular}{|c|c|c|c|c|c|c|}
\hline Characteristic & $\begin{array}{l}\text { Minor complication } \\
\quad(n=174)\end{array}$ & $\begin{array}{l}\text { Major complication } \\
\qquad(n=14)\end{array}$ & P-value & $\mathrm{OR}$ & $95 \% \mathrm{Cl}$ & P-value \\
\hline Age (yr) & & & 0.578 & & & \\
\hline Nonelderly & 103 (59.2) & $7(50.0)$ & & & & \\
\hline Elderly & $71(40.8)$ & $7(50.0)$ & & & & \\
\hline Mean $\pm S D$ & $66.4 \pm 11.9$ & $64.9 \pm 10.7$ & 0.636 & & & \\
\hline Sex & & & 0.580 & & & \\
\hline Male & $93(53.4)$ & $9(64.3)$ & & & & \\
\hline Female & $81(46.6)$ & $5(35.7)$ & & & & \\
\hline Body mass index $\left(\mathrm{kg} / \mathrm{m}^{2}\right)$ & $23.8 \pm 3.2$ & $22.3 \pm 3.3$ & 0.118 & & & \\
\hline Primary tumor location & & & 0.087 & & & 0.222 \\
\hline Colon & $107(61.5)$ & $5(35.7)$ & & 1 & & \\
\hline Rectum & 67 (38.5) & $9(64.3)$ & & 2.252 & $0.612-8.291$ & \\
\hline Operative procedure & & & 0.440 & & & \\
\hline Hemicolectomy & $71(40.8)$ & $4(28.6)$ & & & & \\
\hline Anterior resection & $35(20.1)$ & $2(14.3)$ & & & & \\
\hline LAR/ultra-LAR & $59(33.9)$ & $8(57.1)$ & & & & \\
\hline Colostomy & $9(5.2)$ & $0(0)$ & & & & \\
\hline Open conversion & & & 0.111 & & & \\
\hline No & $168(96.6)$ & $12(85.7)$ & & & & \\
\hline Yes & $6(3.4)$ & $2(14.3)$ & & & & \\
\hline Operative time (min) & $266.2 \pm 102.6$ & $336.6 \pm 117.8$ & 0.015 & 1.003 & 0.998-1.008 & 0.240 \\
\hline Estimated blood loss (mL) & $177.4 \pm 148.1$ & $338.1 \pm 626.2$ & 0.231 & & & \\
\hline Previous operative history & & & 0.549 & & & \\
\hline No & $125(71.8)$ & $9(64.3)$ & & & & \\
\hline Yes & 49 (28.2) & $5(35.7)$ & & & & \\
\hline Smoking history & & & 0.033 & & & \\
\hline No & $125(71.8)$ & $6(42.9)$ & & 1 & & \\
\hline Yes & 49 (28.2) & $8(57.1)$ & & 1.785 & 1.003-3.178 & 0.049 \\
\hline Alcohol history & & & 1.000 & & & \\
\hline No & 119 (68.4) & 10 (71.4) & & & & \\
\hline Yes & 55 (31.6) & $4(28.6)$ & & & & \\
\hline Hypertension & & & 1.000 & & & \\
\hline No & $100(57.5)$ & $8(57.1)$ & & & & \\
\hline Yes & $74(42.5)$ & $6(42.9)$ & & & & \\
\hline Diabetes & & & 0.739 & & & \\
\hline No & $134(77.0)$ & $12(85.7)$ & & & & \\
\hline Yes & $40(23.0)$ & $2(14.3)$ & & & & \\
\hline ASA PS classification & & & 0.506 & & & \\
\hline I & 37 (21.3) & $4(28.6)$ & & & & \\
\hline$\|$ & $118(67.8)$ & $10(71.4)$ & & & & \\
\hline III-IV & 19 (10.9) & $0(0)$ & & & & \\
\hline
\end{tabular}


Table 3. Continued

\begin{tabular}{|c|c|c|c|c|c|c|}
\hline Characteristic & $\begin{array}{l}\text { Minor complication } \\
\quad(n=174)\end{array}$ & $\begin{array}{l}\text { Major complication } \\
\qquad(n=14)\end{array}$ & P-value & $\mathrm{OR}$ & $95 \% \mathrm{Cl}$ & P-value \\
\hline Charlson Comorbidity Index & & & 1.000 & & & \\
\hline 0 & $106(60.9)$ & $9(64.3)$ & & & & \\
\hline 1 & 48 (27.6) & $3(21.4)$ & & & & \\
\hline 2 & $15(8.6)$ & $2(14.3)$ & & & & \\
\hline$\geq 3$ & $5(2.9)$ & $0(0)$ & & & & \\
\hline T stage & & & 0.408 & & & \\
\hline $0-2$ & $72(94.7)$ & $4(28.6)$ & & & & \\
\hline $3-4$ & $102(58.6)$ & $10(71.4)$ & & & & \\
\hline N stage & & & 0.589 & & & \\
\hline 0 & $100(57.5)$ & $7(50.0)$ & & & & \\
\hline $1-2$ & $74(42.5)$ & $7(50.0)$ & & & & \\
\hline Tumor stage & & & 0.467 & & & \\
\hline $0-1$ & $54(31.0)$ & $2(14.3)$ & & & & \\
\hline 2 & $46(26.4)$ & $4(28.6)$ & & & & \\
\hline 3 & $66(37.9)$ & $7(50.0)$ & & & & \\
\hline 4 & $8(4.6)$ & $1(7.1)$ & & & & \\
\hline Histology of primary tumor & & & 0.897 & & & \\
\hline Well & $35(20.1)$ & $2(14.3)$ & & & & \\
\hline Moderately & $125(71.8)$ & $11(78.6)$ & & & & \\
\hline Mucinous or poorly & $14(8.0)$ & $1(7.1)$ & & & & \\
\hline
\end{tabular}

Values are presented as mean \pm standard deviation or number (\%).

OR, odds ratio; Cl, confidence interval; LAR, low anterior resection; ASA PS, American Society of Anesthesiologists physical status.

mended to receive postoperative chemotherapy according to the NCCN guidelines and 90 of 113 patients (79.6\%) received postoperative chemotherapy. Of these, there were 32 elderly patients $(35.6 \%)$ and the mean age was $75.8 \pm 4.5$ years. There were no significant differences in baseline characteristics between the elderly patients who received postoperative chemotherapy and nonelderly patients who received postoperative chemotherapy, except for hypertension, ASA PS classification, and postoperative complication. In the outcomes of postoperative chemotherapy, chemotherapy agents consisting of 5 -fluorouracil, oxaliplatin, and irinotecan were administered in 17 patients (18.9\%), 68 patients (75.6\%), and 5 patients (5.5\%), respectively. There were no significant differences in terms of CTCAE grade, chemotherapy setting, and chemotherapy dose between the elderly and nonelderly patients (Table 4).

Adverse events occurred in 109 cases, including 53 (58.9\%) in grade I, 35 (38.9\%) in grade II, 20 (20.2\%) in grade III, and 1 (1.1\%) in grade IV, respectively. The most common adverse event was mild peripheral sensory neuropathy. Treatment in the intensive care unit was required in 1 patient (1.1\%) because of severe pulmonary embolism. There was no patient chemotherapy-related mortality within 1 year after surgery, whereas 3 patients who did not receive postoperative chemotherapy died because of their
Table 4. Characteristics and outcomes of patients who received postoperative chemotherapy according to the National Comprehensive Cancer Network guidelines $(\mathrm{n}=90)$

\begin{tabular}{lccr}
\hline Characteristic & $\begin{array}{c}\text { Nonelderly } \\
(\mathrm{n}=58)\end{array}$ & $\begin{array}{c}\text { Elderly } \\
(\mathrm{n}=32)\end{array}$ & P-value \\
\hline Age $(\mathrm{yr})$ & $57.2 \pm 8.4$ & $75.8 \pm 4.5$ & $<0.001$ \\
Sex & $29(50.0)$ & $17(53.1)$ & 0.828 \\
$\quad$ Male & $29(50.0)$ & $15(46.9)$ & \\
$\quad$ Female & $23.1 \pm 2.4$ & $23.6 \pm 3.7$ & 0.458 \\
Body mass index $\left(\mathrm{kg} / \mathrm{m}^{2}\right)$ & & & 0.127 \\
Primary tumor location & $30(51.7)$ & $22(68.8)$ & \\
$\quad$ Colon & $28(48.3)$ & $10(31.3)$ & \\
$\quad$ Rectum & & & 0.234 \\
Smoking history & $37(63.8)$ & $25(78.1)$ & \\
$\quad$ No & $21(36.2)$ & $7(21.9)$ & \\
$\quad$ Yes & & & 0.490 \\
Alcohol history & $37(63.8)$ & $23(71.9)$ & \\
$\quad$ No & $21(36.2)$ & $9(28.1)$ & \\
Yes & &
\end{tabular}

(Continued to the next page) 
Table 4. Continued

\begin{tabular}{|c|c|c|c|}
\hline Characteristic & $\begin{array}{l}\text { Nonelderly } \\
(n=58)\end{array}$ & $\begin{array}{l}\text { Elderly } \\
(\mathrm{n}=32)\end{array}$ & P-value \\
\hline Hypertension & & & 0.004 \\
\hline No & $47(81.0)$ & $16(50.0)$ & \\
\hline Yes & $11(19.0)$ & $16(50.0)$ & \\
\hline Diabetes & & & 0.058 \\
\hline No & 50 (86.2) & $22(68.8)$ & \\
\hline Yes & $8(13.8)$ & 10 (31.3) & \\
\hline ASA PS classification & & & 0.001 \\
\hline I & $20(34.5)$ & $1(3.1)$ & \\
\hline$\|$ & $35(60.3)$ & $28(87.5)$ & \\
\hline$\geq \mid \mathrm{II}$ & $3(5.2)$ & $3(9.4)$ & \\
\hline Charlson Comorbidity Index & & & 0.116 \\
\hline 0 & $43(74.1)$ & $17(53.1)$ & \\
\hline 1 & $10(17.2)$ & $11(34.4)$ & \\
\hline$\geq 2$ & $5(8.6)$ & $4(12.5)$ & \\
\hline Tumor stage & & & 0.943 \\
\hline 2 & $16(27.6)$ & $10(31.3)$ & \\
\hline 3 & $37(63.8)$ & $19(59.4)$ & \\
\hline 4 & $5(8.6)$ & $3(9.4)$ & \\
\hline Postoperative complication & & & 0.006 \\
\hline Clavien-Dindo grades $0-I$ & $43(74.1)$ & $14(43.8)$ & \\
\hline Clavien-Dindo grades $\|-\| \|$ & $15(25.9)$ & $18(56.3)$ & \\
\hline Chemotherapy regimen & & & 0.786 \\
\hline 5-Fluorouracil & $10(17.2)$ & $7(21.9)$ & \\
\hline Oxaliplatin & $45(77.6)$ & $23(71.9)$ & \\
\hline Irinotecan & $3(5.2)$ & $2(6.3)$ & \\
\hline Chemotherapy setting & & & 1.000 \\
\hline Adjuvant & 53 (91.4) & $29(90.6)$ & \\
\hline Palliative & $5(8.6)$ & $3(9.4)$ & \\
\hline CTCAE grade & & & 0.288 \\
\hline 0 & 19 (32.8) & $6(18.8)$ & \\
\hline I & $15(25.9)$ & $10(31.3)$ & \\
\hline$\|$ & $14(24.1)$ & $6(18.8)$ & \\
\hline III & $9(15.5)$ & 10 (31.3) & \\
\hline IV & $1(1.7)$ & $0(0)$ & \\
\hline Chemotherapy dose & & & 0.149 \\
\hline Fully dosed & $44(75.9)$ & 19 (59.4) & \\
\hline Dose-reduced & $14(24.1)$ & $13(40.6)$ & \\
\hline Dose reduction (\%) & & & 0.097 \\
\hline 100 & $44(75.9)$ & $19(59.4)$ & \\
\hline$\geq 75$ & $12(20.7)$ & $13(40.6)$ & \\
\hline$\geq 50$ & $2(3.4)$ & $0(0)$ & \\
\hline
\end{tabular}

Values are presented as mean \pm standard deviation or number (\%).

ASA PS, American Society of Anesthesiologists physical status; CTCAE, the Common Terminology Criteria for Adverse Events. comorbid disease (Supplementary Table 2).

Overall adverse events occurred in 40 patients (44.4\%). We found significant differences in overall adverse events according to the ASA PS classification and chemotherapy regimen in univariate analysis. Age was not significantly different between the uneventful course group and overall adverse events group $(\mathrm{P}=$ 0.508). In multivariate analysis, ASA PS classification grade II (reference: ASA grade I; OR, 4.625; 95\% CI, 1.321 to16.187; P= 0.017 ) and oxaliplatin (reference: 5-fluorouracil; OR, 4.767; 95\% CI, 1.168 to $19.451 ; \mathrm{P}=0.029$ ) were independent predictors of overall adverse events of postoperative chemotherapy (Table 5).

Severe adverse events occurred in 20 patients (22.2\%). Compared to mild adverse events, only the chemotherapy regimen was significantly different in univariate analysis. Age was not significantly different between mild and severe adverse events $(\mathrm{P}=$ 0.185 ). In multivariate analysis, only irinotecan (reference: 5-fluorouracil; $\mathrm{OR}, 26.488$; $95 \% \mathrm{CI}, 1.414$ to $496.322 ; \mathrm{P}=0.028$ ) was an independent predictor of severe adverse events of postoperative chemotherapy (Table 6).

Among the 90 patients who received postoperative chemotherapy, postoperative chemotherapy could not be completed in 11 patients $(12.2 \%)$ because of severe adverse event in 4 patients, the patient's refusal to continue chemotherapy (in the absence of chemotherapy-related complications) in 6 patients, and follow-up loss in 1 patient (Supplementary Table 3). Only age was significantly different between patients who completed or stopped postoperative chemotherapy. There were no significant differences in terms of chemotherapy regimen, chemotherapy setting, dose reduction, and adverse events between the 2 groups. In multivariate analysis, only age was significantly associated with stopping postoperative chemotherapy (OR, 12.317; 95\% CI, 2.390 to 63.491; $\mathrm{P}=0.003)$ (Table 7).

\section{DISCUSSION}

This study demonstrates the effect of age on laparoscopic surgery and postoperative chemotherapy in consecutive patients with CRC. We evaluated postoperative complications and the adverse events of postoperative chemotherapy by using the $\mathrm{CD}$ classification and the CTCAE version 5.0, respectively, in elderly patients compared to nonelderly patients. Our results indicated that major postoperative complications were not different between elderly and nonelderly patients with CRC, although the incidence of overall postoperative complications was higher in the elderly group. In addition, overall and severe adverse events of postoperative chemotherapy were not different between elderly and nonelderly patients, whereas chronologic age was highly associated with factors influencing the termination of postoperative chemotherapy.

There is still no general consensus on the definition of elderly. In a recent review article regarding older age life expectancy, the World Health Organization defined older adults as those aged 60 
Table 5. Univariate and multivariate analysis of factors affecting overall adverse events of postoperative chemotherapy ( $\mathrm{n}=90$ )

\begin{tabular}{|c|c|c|c|c|c|c|}
\hline Characteristic & Uneventful course $(n=50)$ & Overall adverse event $(n=40)$ & P-value & OR & $95 \% \mathrm{Cl}$ & P-value \\
\hline Age (yr) & & & 0.508 & & & \\
\hline Nonelderly & $34(68.0)$ & $24(60.0)$ & & & & \\
\hline Elderly & $16(32.0)$ & $16(40.0)$ & & & & \\
\hline Mean age (yr) & $62.0 \pm 13.4$ & $66.0 \pm 8.3$ & 0.090 & & & \\
\hline Nonelderly & $54.7 \pm 9.4$ & $60.6 \pm 5.2$ & 0.004 & & & \\
\hline Elderly & $77.6 \pm 3.7$ & $74.1 \pm 4.6$ & 0.027 & & & \\
\hline Sex & & & 0.144 & & & \\
\hline Male & $22(44.0)$ & $24(60.0)$ & & & & \\
\hline Female & $28(56.0)$ & $16(40.0)$ & & & & \\
\hline Body mass index $\left(\mathrm{kg} / \mathrm{m}^{2}\right)$ & $22.9 \pm 2.9$ & $23.7 \pm 2.9$ & 0.246 & & & \\
\hline Primary tumor location & & & 0.203 & & & \\
\hline Colon & $32(64.0)$ & $20(50.0)$ & & & & \\
\hline Rectum & $18(36.0)$ & $20(50.0)$ & & & & \\
\hline Smoking history & & & 0.115 & & & \\
\hline No & $38(76.0)$ & $24(60.0)$ & & & & \\
\hline Yes & $12(24.0)$ & $16(40.0)$ & & & & \\
\hline Alcohol history & & & 0.265 & & & \\
\hline No & $36(72.0)$ & $24(60.0)$ & & & & \\
\hline Yes & $14(28.0)$ & $16(40.0)$ & & & & \\
\hline Hypertension & & & 0.652 & & & \\
\hline No & $36(72.0)$ & $27(67.5)$ & & & & \\
\hline Yes & $14(28.0)$ & $13(32.5)$ & & & & \\
\hline Diabetes & & & 0.061 & & & 0.396 \\
\hline No & $44(88.0)$ & $28(70.0)$ & & 1 & & \\
\hline Yes & $6(12.0)$ & $12(30.0)$ & & 1.683 & $0.505-5.605$ & \\
\hline ASA PS classification & & & 0.018 & & & 0.057 \\
\hline । & $17(34.0)$ & $4(10.0)$ & & 1 & & \\
\hline$\|$ & $31(62.0)$ & $32(80.0)$ & & 4.625 & $1.321-16.187$ & 0.017 \\
\hline$\geq|l|$ & $2(4.0)$ & $4(10.0)$ & & 4.098 & $0.438-38.303$ & 0.216 \\
\hline Charlson Comorbidity Index & & & 0.260 & & & \\
\hline 0 & $37(74.0)$ & $23(57.5)$ & & & & \\
\hline 1 & $9(18.0)$ & $12(30.0)$ & & & & \\
\hline$\geq 2$ & $4(8.0)$ & $5(12.5)$ & & & & \\
\hline Chemotherapy regimen & & & 0.014 & & & 0.054 \\
\hline 5-Fluorouracil & $14(28.0)$ & $3(7.5)$ & & 1 & & \\
\hline Oxaliplatin & $35(70.0)$ & $33(82.5)$ & & 4.767 & $1.168-19.451$ & 0.029 \\
\hline Irinotecan & $1(2.0)$ & $4(10.0)$ & & 13.531 & $0.977-187.293$ & 0.052 \\
\hline Chemotherapy setting & & & 0.132 & & & \\
\hline Adjuvant & $48(96.0)$ & $34(85.0)$ & & & & \\
\hline Palliative & $2(4.0)$ & $6(15.0)$ & & & & \\
\hline
\end{tabular}

Values are presented as mean \pm standard deviation or number (\%).

OR, odds ratio; Cl, confidence interval; ASA PS, American Society of Anesthesiologists physical status. 
Table 6. Univariate and multivariate analysis of factors affecting severe adverse events of postoperative chemotherapy ( $\mathrm{n}=90)$

\begin{tabular}{|c|c|c|c|c|c|c|}
\hline Characteristic & Mild adverse event $(n=70)$ & Severe adverse event $(n=20)$ & P-value & OR & $95 \% \mathrm{Cl}$ & P-value \\
\hline Age (yr) & & & 0.185 & & & \\
\hline Nonelderly & $48(68.6)$ & $10(50.0)$ & & & & \\
\hline Elderly & $22(31.4)$ & $10(50.0)$ & & & & \\
\hline Mean age (yr) & $64.3 \pm 11.9$ & $68.0 \pm 8.4$ & 0.084 & 1.047 & $0.985-1.113$ & 0.144 \\
\hline Nonelderly & $56.1 \pm 8.6$ & $62.3 \pm 4.8$ & 0.032 & & & \\
\hline Elderly & $75.9 \pm 4.3$ & $75.8 \pm 5.1$ & 0.971 & & & \\
\hline Sex & & & 0.450 & & & \\
\hline Male & $34(48.6)$ & $12(60.0)$ & & & & \\
\hline Female & $36(51.4)$ & $8(40.0)$ & & & & \\
\hline Body mass index $\left(\mathrm{kg} / \mathrm{m}^{2}\right)$ & $23.0 \pm 2.8$ & $24.1 \pm 3.1$ & 0.122 & & & \\
\hline Primary tumor location & & & 0.802 & & & \\
\hline Colon & $41(58.6)$ & $11(55.0)$ & & & & \\
\hline Rectum & $29(41.4)$ & $9(45.0)$ & & & & \\
\hline Smoking history & & & 0.785 & & & \\
\hline No & $49(70.0)$ & $13(65.0)$ & & & & \\
\hline Yes & $21(30.0)$ & $7(35.0)$ & & & & \\
\hline Alcohol history & & & 0.592 & & & \\
\hline No & $48(68.6)$ & $12(60.0)$ & & & & \\
\hline Yes & $22(31.4)$ & $8(40.0)$ & & & & \\
\hline Hypertension & & & 0.783 & & & \\
\hline No & $48(68.6)$ & $15(75.0)$ & & & & \\
\hline Yes & $22(31.4)$ & $5(25.0)$ & & & & \\
\hline Diabetes & & & 0.536 & & & \\
\hline No & $57(81.4)$ & $15(75.0)$ & & & & \\
\hline Yes & $13(18.6)$ & $5(25.0)$ & & & & \\
\hline ASA PS classification & & & 0.056 & & & 0.418 \\
\hline । & $20(28.6)$ & $1(5.0)$ & & 1 & & \\
\hline$\|$ & $46(65.7)$ & $17(85.0)$ & & 3.795 & $0.414-34.784$ & 0.238 \\
\hline$\geq|l|$ & $4(5.7)$ & $2(10.0)$ & & 1.701 & $0.068-42.696$ & 0.747 \\
\hline Charlson Comorbidity Index & & & 0.579 & & & \\
\hline 0 & $47(67.1)$ & $13(65.0)$ & & & & \\
\hline 1 & $15(21.4)$ & $6(30.0)$ & & & & \\
\hline$\geq 2$ & $8(11.4)$ & $1(5.0)$ & & & & \\
\hline Chemotherapy regimen & & & 0.015 & & & 0.082 \\
\hline 5-Fluorouracil & $14(20.0)$ & $3(15.0)$ & & 1 & & \\
\hline Oxaliplatin & 55 (78.6) & $13(65.0)$ & & 1.536 & $0.353-6.678$ & 0.567 \\
\hline Irinotecan & $1(1.4)$ & $4(20)$ & & 26.488 & $1.414-496.322$ & 0.028 \\
\hline Chemotherapy setting & & & 0.369 & & & \\
\hline Adjuvant & $65(92.9)$ & $17(85.0)$ & & & & \\
\hline Palliative & $5(7.1)$ & $3(15.0)$ & & & & \\
\hline
\end{tabular}

Values are presented as mean \pm standard deviation or number (\%).

OR, odds ratio; Cl, confidence interval; ASA PS, American Society of Anesthesiologists physical status. 
Table 7. Univariate and multivariate analysis factors associated with stopping postoperative chemotherapy in patients who received postoperative chemotherapy $(n=90)$

\begin{tabular}{|c|c|c|c|c|c|c|}
\hline Characteristic & Complete chemotherapy $(n=79)$ & $\begin{array}{l}\text { Stopped chemotherapy } \\
\qquad(\mathrm{n}=11)\end{array}$ & P-value & $\mathrm{OR}$ & $95 \% \mathrm{Cl}$ & P-value \\
\hline Age (yr) & & & 0.001 & & & \\
\hline Nonelderly & $56(70.9)$ & $2(18.2)$ & & 1 & & \\
\hline Elderly & $23(29.1)$ & $9(81.8)$ & & 12.317 & $2.390-63.491$ & 0.003 \\
\hline Mean age (yr) & $62.3 \pm 11.1$ & $74.7 \pm 9.1$ & 0.001 & & & \\
\hline Nonelderly & $57.1 \pm 8.5$ & $59.0 \pm 5.6$ & 0.755 & & & \\
\hline Elderly & $74.9 \pm 4.1$ & $78.2 \pm 4.8$ & 0.059 & & & \\
\hline Sex & & & 0.523 & & & \\
\hline Male & $39(49.4)$ & $7(63.6)$ & & & & \\
\hline Female & $40(50.6)$ & $4(36.4)$ & & & & \\
\hline Body mass index $\left(\mathrm{kg} / \mathrm{m}^{2}\right)$ & $23.4 \pm 2.9$ & $21.8 \pm 2.3$ & 0.085 & 0.818 & $0.654-1.022$ & 0.077 \\
\hline Primary tumor location & & & 0.754 & & & \\
\hline Colon & $45(57.0)$ & $7(63.6)$ & & & & \\
\hline Rectum & $34(43.0)$ & $4(36.4)$ & & & & \\
\hline Smoking history & & & 1.000 & & & \\
\hline No & $54(68.4)$ & $8(72.7)$ & & & & \\
\hline Yes & $25(31.6)$ & $3(27.3)$ & & & & \\
\hline Alcohol history & & & 0.746 & & & \\
\hline No & $52(65.8)$ & $8(72.7)$ & & & & \\
\hline Yes & $27(34.2)$ & $3(27.3)$ & & & & \\
\hline Hypertension & & & 0.728 & & & \\
\hline No & $56(70.9)$ & $7(63.6)$ & & & & \\
\hline Yes & $23(29.1)$ & $4(36.4)$ & & & & \\
\hline Diabetes & & & 0.687 & & & \\
\hline No & $64(81.0)$ & $8(72.7)$ & & & & \\
\hline Yes & $15(19.0)$ & $3(27.3)$ & & & & \\
\hline ASA PS classification & & & 0.454 & & & \\
\hline I & $20(25.3)$ & $1(9.1)$ & & & & \\
\hline$\|$ & $54(85.7)$ & $9(81.8)$ & & & & \\
\hline$\geq \mathrm{III}$ & $5(6.3)$ & $1(9.1)$ & & & & \\
\hline Charlson Comorbidity Index & & & 0.884 & & & \\
\hline 0 & $53(67.1)$ & $7(63.6)$ & & & & \\
\hline 1 & $18(22.8)$ & $3(27.3)$ & & & & \\
\hline$\geq 2$ & $8(10.1)$ & $1(9.1)$ & & & & \\
\hline Chemotherapy regimen & & & 0.742 & & & \\
\hline 5-Fluorouracil & $15(19.0)$ & $2(18.2)$ & & & & \\
\hline Oxaliplatin & $59(74.7)$ & $9(81.8)$ & & & & \\
\hline Irinotecan & $5(6.3)$ & $0(0)$ & & & & \\
\hline Chemotherapy setting & & & 0.589 & & & \\
\hline Adjuvant & $71(89.9)$ & $11(13.4)$ & & & & \\
\hline Palliative & $8(100)$ & $0(0)$ & & & & \\
\hline
\end{tabular}


Table 7. Continued

\begin{tabular}{|c|c|c|c|c|c|c|}
\hline Characteristic & Complete chemotherapy $(n=79)$ & $\begin{array}{l}\text { Stopped chemotherapy } \\
\qquad(n=11)\end{array}$ & P-value & $\mathrm{OR}$ & $95 \% \mathrm{Cl}$ & P-value \\
\hline Adverse events & & & 0.254 & & & \\
\hline CTCAE grades 0-॥ & $63(79.7)$ & $7(63.6)$ & & & & \\
\hline CTCAE grades III-IV & $16(20.3)$ & $4(34.6)$ & & & & \\
\hline Chemotherapy dose & & & 0.295 & & & \\
\hline Fully dosed & 57 (72.2) & $6(54.5)$ & & & & \\
\hline Dose-reduced & $22(27.8)$ & $5(45.5)$ & & & & \\
\hline Dose reduction (\%) & & & 0.447 & & & \\
\hline 100 & 57 (72.2) & $6(54.5)$ & & & & \\
\hline$\geq 75$ & $20(25.3)$ & $5(45.5)$ & & & & \\
\hline$\geq 50$ & $2(2.5)$ & $0(0)$ & & & & \\
\hline
\end{tabular}

Values are presented as mean \pm standard deviation or number (\%).

OR, odds ratio; Cl, confidence interval; LAR, low anterior resection; ASA PS, American Society of Anesthesiologists physical status; CTCAE, the Common Terminology Criteria for Adverse Events.

years or older [18]. In contrast, several studies defined the elderly as 80 years-of-age and older, which may be more appropriate given the increase in the average life expectancy [19]. However, many clinical studies regarding the clinical features and prognosis of elderly patients with CRC defined elderly patients as those over 70 or 75 years old [20]. Therefore, we defined 70 years of age as the threshold between nonelderly and elderly, by considering the mean age of $66.3 \pm 11.8$ in the present study.

Previous studies have suggested that age is not an independent predictor of morbidity and mortality in laparoscopic CRC surgery $[21,22]$, although several studies showed that increased age increased the risk of postoperative complications [23, 24]. In fact, elderly patients naturally have more comorbidities and are more sensitive to surgical stress than nonelderly patients, which might affect the overall complication rates. A recent meta-analysis reported that the incidence of overall postoperative complications was slightly higher in elderly patients than in nonelderly patients, whereas there was no difference between elderly and nonelderly patients in anastomotic leakage and mortality [20]. In the present study, overall postoperative complication rates after laparoscopic CRC surgery did differ between the elderly and nonelderly patients (54.4\% vs. $45.6 \%$ ), because more postoperative complications with $\mathrm{CD}$ grade II requiring pharmacological treatment ( $38.5 \%$ vs. $21.8 \%$ ) occurred in the elderly patients. However, there was no difference between the 2 groups in major postoperative complications requiring invasive treatment in this study, as in the meta-analysis. Therefore, we suggest that laparoscopic CRC surgery in elderly patients is feasible, although careful management is required after surgery in elderly patients depending on their intrinsic nature.

Several factors, including male gender, comorbidities, emergency procedures, long duration of operation, and distant metastasis, were reported to be predictive factors for major postopera- tive complications in CRC patients, although this finding is controversial $[23,24]$. The current study showed that only smoking history was associated with major postoperative complications after laparoscopic CRC surgery. Smoking is known to cause microvascular disease, tissue ischemia, and hypoxia, which might be contributing factors to poor anastomotic healing [25]. In a study of over 47,000 patients, Sharma et al. [26] demonstrated that smoking increases the risk of major postoperative complications after all types of colorectal surgery. Our findings correlated with previous studies indicating an increased risk of major postoperative complications, although the effect of smoking on major postoperative complications was not fully understood. Therefore, a well-designed prospective study would be needed to validate the findings in this study.

Previous studies suggested that the incidence of toxic effects for postoperative chemotherapy was not increased among elderly patients with CRC $[9,10]$, although it remains a controversial issue [27]. In the real-world setting, patient age strongly influenced the recommendations for chemotherapy by both surgeons and oncologists [28]. This might be attributed to the degeneration of liver or kidney function seen more often in elderly patients, the risk of which could increase with treatment. However, for fit elderly patients, no significant interaction was observed between age and treatment efficacy and postoperative chemotherapy appeared to achieve similar survival benefits with no greater toxicity than that seen in nonelderly patients [11]. The present study indicated that age was not an independent predictor of overall and severe adverse events of postoperative chemotherapy in patients with CRC, probably because elderly patients had similar comorbidities and slightly higher ASA physical status classification. These findings suggest that postoperative chemotherapy is feasible in elderly patients who may be good candidates.

Although some studies reported that tumor types and chemo- 
therapy regimen were associated with chemotherapy toxicity [29], the results remain controversial because of the heterogeneity of selection criteria and variations in multimodal treatment. In the current study, chemotherapy regimen was associated with overall and severe adverse events after postoperative chemotherapy in patients with CRC; the risk of overall and severe adverse events was higher in patients receiving chemotherapy consisting of oxaliplatin and irinotecan, respectively. However, the number of patients in the irinotecan group was too low in this study. Therefore, these findings should be confirmed in larger studies.

There were various studies identifying factors to discontinue postoperative chemotherapy [29]. We focused on age and the adverse events of postoperative chemotherapy as factors that stopped chemotherapy. Our results revealed that only age was significantly associated with the termination of postoperative chemotherapy, whereas the severity of adverse events related to chemotherapy did not differ between patients who completed or stopped postoperative chemotherapy. We thought that selection bias might have been present because patients who potentially could not tolerate postoperative chemotherapy were already excluded from receiving chemotherapy by the treatment recommendation process. Nevertheless, more than half of the patients who stopped postoperative chemotherapy could not complete postoperative chemotherapy because of reasons other than the side effects of chemotherapy which included old age, economic difficulties, lack of family support, and psychological problems. In particular, 4 of the 9 elderly patients (44.4\%) who stopped postoperative chemotherapy discontinued treatment because of old age without any adverse events. Therefore, we suggest that patients and family members should receive education on the importance of establishing treatment based on patient disease stage and physiologic status, rather than chronologic age.

This study had some limitations. First, there was a potential for selection bias, compounded by the retrospective design and single-center study. In the present study, the number of males was lower than the number of females in the elderly patient group. One of the reasons for the lower number of males is because the mean lifespan of males is approximately 10 years shorter than that of females [18]. Therefore, patient baseline characteristics could be different and might have affected the outcomes of laparoscopic CRC surgery and postoperative chemotherapy. Secondly, the long-term outcomes for laparoscopic CRC surgery were not assessed. Previous studies have reported that the long-term outcomes did not differ between younger and elderly patients [30]. Thirdly, the number of patients that stopped postoperative chemotherapy was relatively small because patients who might have stopped chemotherapy were already excluded during the treatment recommendation process. Therefore, further large-scale prospective studies will be helpful to verify our results.

In conclusion, this study shows that laparoscopic CRC surgery and postoperative chemotherapy were feasible in elderly patients, whereas age was still considered a factor in the decision to stop postoperative chemotherapy. Therefore, we suggest that further efforts are needed to ensure that elderly patients have the opportunity to make informed decisions regarding postoperative chemotherapy.

\section{CONFLICT OF INTEREST}

No potential conflict of interest relevant to this article was reported.

\section{SUPPLEMENTARY MATERIALS}

Supplementary Tables 1-3 can be found via https://doi.org/10.3393/ ac.2019.10.03.

\section{REFERENCES}

1. Surgery for colorectal cancer in elderly patients: a systematic review. Colorectal Cancer Collaborative Group. Lancet 2000;356: 968-74.

2. Arnold M, Sierra MS, Laversanne M, Soerjomataram I, Jemal A, Bray F. Global patterns and trends in colorectal cancer incidence and mortality. Gut 2017;66:683-91.

3. Lacy AM, Garcia-Valdecasas JC, Delgado S, Castells A, Taura P, Pique JM, et al. Laparoscopy-assisted colectomy versus open colectomy for treatment of non-metastatic colon cancer: a randomised trial. Lancet 2002;359:2224-9.

4. Kang SB, Park JW, Jeong SY, Nam BH, Choi HS, Kim DW, et al. Open versus laparoscopic surgery for mid or low rectal cancer after neoadjuvant chemoradiotherapy (COREAN trial): short-term outcomes of an open-label randomised controlled trial. Lancet Oncol 2010;11:637-45.

5. Clinical Outcomes of Surgical Therapy Study Group, Nelson H, Sargent DJ, Wieand HS, Fleshman J, Anvari M, et al. A comparison of laparoscopically assisted and open colectomy for colon cancer. N Engl J Med 2004;350:2050-9.

6. Zollinger A, Krayer S, Singer T, Seifert B, Heinzelmann M, Schlumpf R, et al. Haemodynamic effects of pneumoperitoneum in elderly patients with an increased cardiac risk. Eur J Anaesthesiol 1997;14:266-75.

7. Al-Refaie WB, Parsons HM, Habermann EB, Kwaan M, Spencer MP, Henderson WG, et al. Operative outcomes beyond 30-day mortality: colorectal cancer surgery in oldest old. Ann Surg 2011; 253:947-52.

8. National Comprehensive Cancer Network. NCCN clinical practice guidelines in oncology (NCCN Guidelines): rectal cancer (version 3.2018) [Internet]. Fort Wathington (PA): National Comprehensive Cancer Network; 2018 [cited 2019 Feb 6]. Available from: https://www.nccn.org/professionals/physician_gls/pdf/ rectal.pdf.

9. Balducci L, Extermann M. Management of cancer in the older person: a practical approach. Oncologist 2000;5:224-37. 
10. Sargent DJ, Goldberg RM, Jacobson SD, Macdonald JS, Labianca R, Haller DG, et al. A pooled analysis of adjuvant chemotherapy for resected colon cancer in elderly patients. N Engl J Med 2001; 345:1091-7.

11. Papamichael D, Audisio R, Horiot JC, Glimelius B, Sastre J, Mitry E, et al. Treatment of the elderly colorectal cancer patient: SIOG expert recommendations. Ann Oncol 2009;20:5-16.

12. Ananda S, Field KM, Kosmider S, Compston D, Desai J, Lim LC, et al. Patient age and comorbidity are major determinants of adjuvant chemotherapy use for stage III colon cancer in routine clinical practice. J Clin Oncol 2008;26:4516-7.

13. Lee YH, Oh HK, Kim DW, Ihn MH, Kim JH, Son IT, et al. Use of a comprehensive geriatric assessment to predict short-term postoperative outcome in elderly patients with colorectal cancer. Ann Coloproctol 2016;32:161-9.

14. Clavien PA, Barkun J, de Oliveira ML, Vauthey JN, Dindo D, Schulick RD, et al. The Clavien-Dindo classification of surgical complications: five-year experience. Ann Surg 2009;250:187-96.

15. Mrak K, Eberl T, Laske A, Jagoditsch M, Fritz J, Tschmelitsch J. Impact of postoperative complications on long-term survival after resection for rectal cancer. Dis Colon Rectum 2013;56:20-8.

16. The U.S. Department of Health \& Human Services. Common terminology criteria for adverse events (CTCAE) version 5.0. Washington (DC): The U.S. Department of Health \& Human Services; 2017 [cited 2019 Feb 6]. Available from: https://ctep. cancer.gov/protocolDevelopment/electronic_applications/docs/ CTCAE_v5_Quick_Reference_5x7.pdf.

17. Hurria A, Togawa K, Mohile SG, Owusu C, Klepin HD, Gross CP, et al. Predicting chemotherapy toxicity in older adults with cancer: a prospective multicenter study. J Clin Oncol 2011;29:3457-65.

18. Mathers CD, Stevens GA, Boerma T, White RA, Tobias MI. Causes of international increases in older age life expectancy. Lancet 2015;385:540-8.

19. Li Y, Wang S, Gao S, Yang C, Yang W, Guo S. Laparoscopic colorectal resection versus open colorectal resection in octogenarians: a systematic review and meta-analysis of safety and efficacy. Tech Coloproctol 2016;20:153-62.

20. Hoshino N, Fukui Y, Hida K, Sakai Y. Short-term outcomes of laparoscopic surgery for colorectal cancer in the elderly versus non-elderly: a systematic review and meta-analysis. Int J Colorectal Dis 2019;34:377-86.
21. Kuhry E, Schwenk WF, Gaupset R, Romild U, Bonjer HJ. Longterm results of laparoscopic colorectal cancer resection. Cochrane Database Syst Rev 2008;(2):CD003432.

22. Seishima R, Okabayashi K, Hasegawa H, Tsuruta M, Shigeta K, Matsui S, et al. Is laparoscopic colorectal surgery beneficial for elderly patients? A systematic review and meta-analysis. J Gastrointest Surg 2015;19:756-65.

23. Alves A, Panis Y, Mathieu P, Mantion G, Kwiatkowski F, Slim K; Association Francaise de Chirurgie. Postoperative mortality and morbidity in French patients undergoing colorectal surgery: results of a prospective multicenter study. Arch Surg 2005;140:27883.

24. Kirchhoff P, Dincler S, Buchmann P. A multivariate analysis of potential risk factors for intra- and postoperative complications in 1316 elective laparoscopic colorectal procedures. Ann Surg 2008; 248:259-65.

25. Alberg AJ, Shopland DR, Cummings KM. The 2014 Surgeon General's report: commemorating the 50th Anniversary of the 1964 Report of the Advisory Committee to the US Surgeon General and updating the evidence on the health consequences of cigarette smoking. Am J Epidemiol 2014;179:403-12.

26. Sharma A, Deeb AP, Iannuzzi JC, Rickles AS, Monson JR, Fleming FJ. Tobacco smoking and postoperative outcomes after colorectal surgery. Ann Surg 2013;258:296-300.

27. Laurent M, Des Guetz G, Bastuji-Garin S, Culine S, Caillet P, Aparicio T, et al. Chronological age and risk of chemotherapy nonfeasibility: a real-life cohort study of 153 stage II or III colorectal cancer patients given adjuvant-modified FOLFOX6. Am J Clin Oncol 2018;41:73-80.

28. Jorgensen ML, Young JM, Solomon MJ. Older patients and adjuvant therapy for colorectal cancer: surgeon knowledge, opinions, and practice. Dis Colon Rectum 2011;54:335-41.

29. van Abbema DL, van den Akker M, Janssen-Heijnen ML, van den Berkmortel F, Hoeben A, de Vos-Geelen J, et al. Patient- and tumor-related predictors of chemotherapy intolerance in older patients with cancer: a systematic review. J Geriatr Oncol 2019;10:3141.

30. Lim SW, Kim YJ, Kim HR. Laparoscopic surgery for colorectal cancer in patients over 80 years of age: the morbidity outcomes. Ann Surg Treat Res 2017;92:423-8. 